\title{
High Molecular Weight Polymers. Simple Polymerization of Methacrylic Monomers Using $\left(\mathrm{Ph}_{2} \mathbf{P C l} /\right.$ Epoxide) as Pre-Initiator
}

\author{
Ren-Hua Jin, ${ }^{\dagger}$ Yoshihumi Hanazaki, and Tadatomi Nishikubo* \\ Department of Materials Science, Faculty of Engineering, Miyazaki University, \\ Miyazaki 889-21, Japan \\ * Department of Applied Chemistry, Faculty of Engineering, Kanagawa University, \\ Kanagawa-ku, Yokohama 221, Japan
}

(Received April 18, 1996)

\begin{abstract}
Chlorodiphenylphosphine (CDP) was used as a pre-initiator in the polymerization of methacrylates having methyl, benzyl, $n$-butyl, $t$-butyl, cyclohexyl, and $n$-hexyl substituents in the presence of catalytic amounts of epoxides. This polymerization system was influenced by solvents and epoxides structure, and polymer molecular weight and yield were dependent strongly on the alkyl group of ester of monomers. Under bulk conditions, the polymerization of methacrylates afforded easily high molecular weight polymers $\left(M_{n}\right.$ over five million) with relative narrow polydispersity (1.2-1.5). Based on several experimental results, it is discussed that the pre-initiator reacts with monomer forming phosphinoenolate from which the polymerization proceeds with the group transfer mechanism.

KEY WORDS Chlorodiphenylphosphine / Epoxides / Methacrylates / High Molecular Weight Polymers / Group Transfer Polymerization / Pre-Initiator /
\end{abstract}

The development of new initiator systems for polymerization of (meth)acrylates is currently interested in the synthetic polymer chemistry. In recent years, some well defined initiator systems such as organosilicon, ${ }^{1}$ organolanthanide, ${ }^{2}$ and aluminum porphyrins ${ }^{3}$ were established, with which molecular weight, molecular dispersion, and block chain are controlled. In numerous anionic or other controlled living polymerizations of (meth)acrylic monomers, few results are related to the synthesis of high molecular weight polymers $\left(M_{n}>10^{5}\right) .{ }^{2,3}$ With organolanthanide initiator systems, the synthesis of poly(methyl methacrylate) not only with high molecular weight but also with nearly mono-dispersion has been conducted by Yasuda and co-workers. ${ }^{2}$ However, particular care and skill are usually required in the polymerization using organometal compounds as initiators. Group transfer polymerization ${ }^{1}$ using organosilicon initiator is a relatively convenient method for controlled polymerization, although this method does not result in high molecular weight polymers. On the other hand, phosphorous compounds are also candidates for initiators. As reported by Hatada group, tertiary phosphines are usable initiators ${ }^{4}$ in the presence of triethylaluminum by which highly syndiotactic polymethacrylates can be produced. Recently, we reported that a catalytic amount of chlorodiphenylphosphine (CDP) induces polymerization $^{5}$ of (meth)acrylates monomers in the presence of catalytic amount of epoxides. Different with cyclic phenyl phosphonite used as a comonomer for zwitterionic polymerization with $\alpha, \beta$-unsaturated carbonyl compounds, ${ }^{6-8}$ (CDP/epoxide) can be used as an initiator for polymerization of (meth)acrylic monomers. This paper reports a simple method to prepare high molecular weight methacrylic polymers with relatively lower polydispersities by using (CDP/epoxide) as pre-initiator.

\footnotetext{
† To whom correspondence should be addressed.
}

\section{EXPERIMENTAL}

Reagents

All monomers, methyl methacrylate (MMA), $n$-butyl methacrylate ( $n$-BMA), tert-butyl methacrylate ( $t$-BMA), benzyl methacrylate (BzMA), $n$-hexyl methacrylate (n-HMA), cyclohexyl methacrylate (c-HMA), glycidyl methacrylate (GMA), ethyl acrylate (EA), and $n$-butyl acrylate $(n-\mathrm{BA})$ were purchased from Tokyo Kasei Inc. and used after passing through a silica column and then drying on $4 \AA$ molecular sieves. Diglyme and dimethyl formamide (DMF) were distilled from $\mathrm{CaH}_{2}$ under reduced pressure. The other solvents were distilled from $\mathrm{CaH}_{2}$ or sodium by usual methods. CDP was used without further purification.

\section{Typical Bulk Polymerization of n-BMA}

A $50 \mathrm{ml}$, one necked round bottomed flask, fitted with magnetic stirring bar, and three-way cock, was flushed with argon under reduced pressure and heated condition. While flushing argon, $n$-butyl methacrylate $(14.5 \mathrm{mmol}$, $2.3 \mathrm{ml}), \mathrm{CDP}\left(0.09 \mathrm{mmol}, 0.1 \mathrm{ml}\right.$ from $0.2 \mathrm{~g} \mathrm{ml}^{-1}$ toluene solution), and then epoxide (GMA, $0.01 \mathrm{ml}$ ) were added to the flask with syringes, respectively. The mixture was stirred for $24 \mathrm{~h}$ at $60^{\circ} \mathrm{C}$. The polymerized product dissolved in chloroform was poured into methanol and the precipitation was washed with methanol and dried. Yield of poly(n-BMA): $80 \%$.

\section{Typical Solution Polymerization of MMA}

A $50 \mathrm{ml}$, one necked round bottomed flask, fitted with magnetic stirring bar, and three-way cock, was flushed with argon under reduced pressure and heated condition. While flushing argon, methyl methacrylate $(14.5 \mathrm{mmol})$, CDP $\left(0.09 \mathrm{mmol}, 0.1 \mathrm{ml}\right.$ from $0.2 \mathrm{~g} \mathrm{ml}^{-1}$ toluene solution), DMF (10.5 ml), and then epoxide (GMA, $0.01 \mathrm{ml})$ were added to the flask. The solution was stirred for $24 \mathrm{~h}$ at $60^{\circ} \mathrm{C}$. The polymerized product diluted with chloroform was poured into methanol and precipitation was 
washed with methanol and dried. Yield of poly(MMA): $80 \%$.

\section{Polymer Characterization}

GPC measurements were performed on Tosoh GPC system using THF as the eluent. Molecular weight and molecular weight distribution were calculated on the basis of the calibrated with polystyrene standards. Triad tacticities for poly(MMA) were determined with ${ }^{1} \mathrm{H}$ NMR $\left(250 \mathrm{MHz}\right.$, in $\left.\mathrm{CDCl}_{3}\right)$ from the integration of $\alpha$-methyl protons $(0.7-1.3 \mathrm{ppm}){ }^{9}$

\section{RESULTS}

In our previous work, it was observed that CDP reacts rapidly with epoxy compounds yielding $1: 1$ adduct $^{10}$ as these two compounds were mixed in equimolar ratio in bulk and that CDP also reacts quickly with vinyl methyl ketone to give $1: 1$ adduct. $^{11}$ When a catalytic amount of CDP was added to GMA, polymerization occurred rapidly to give poly(GMA). ${ }^{5}$ We concluded that the combination of CDP and epoxide is a useful initiator for polymerization of (meth)acrylates. Considering the mechanism (discussed in the later) of this polymerization which takes place after forming an enolate (which may be an intermediate formed from CDP and monomer), we define here that CDP is a pre-initiator.

With MMA as monomer, the effects of concentration of MMA, solvents, epoxides, and temperature on the polymerization of MMA were studied. Table I shows the effects of concentrations of MMA on polymerizations. In these polymerizations, MMA (14.5 mmol) was diluted by increasing solvent amount from 0.5 to $10 \mathrm{ml}$. The yield of poly(MMA) decreased with increasing concentration. Unexpectedly, the molecular weight of poly(MMA) increased nearly 10 times as concentration increased from 1.2 to $3.6 \mathrm{moll}^{-1}$ indicating that the $\mathrm{CDP} /$ epoxide pre-initiator favors to give high molecular weight polymer at high monomer concentration. In addition, the molecular weight of the poly(MMA) increased when the ratio of monomer to CDP increased. Also, the number average molecular weight of poly(MMA) increased to $504.3 \times 10^{3}$ from $253.7 \times 10^{3}$ as reaction time was extended to $24 \mathrm{~h}$ from $6 \mathrm{~h}$. In solution polymerization, the solvents including polar and nonpolar one were used at $[\mathrm{MMA}] /[\mathrm{CDP}]=160$, epoxide $(\mathrm{GMA})=0.01 \mathrm{ml}, 60^{\circ} \mathrm{C}$, and $24 \mathrm{~h}$ conditions. The polymerization of MMA proceeded smoothly in DMF and diglyme, but not in acetone, acetonitrile, DMSO, toluene, xylene, or $\mathrm{CCl}_{4}$ (see Table II). The same was seen in the polymerizations of acrylates such as EA, n-BA. The polymerization of using CDP/epoxide as pre-initiator may thus be strongly dependent on solvent. In the solution polymerization, DMF is a most suitable solvent.

The epoxides play an important roles in this polymerization system. ${ }^{5}$ Styrene oxide (SO), cyclohexene oxide (CHO), and GMA promoted effectively the polymerization of MMA. However, other examined epoxides, such as chloromethyl oxirane, glycidyl phenyl ether, propylene oxide, 1-methoxy-2-methylpropylene oxide, etc., were not efficient to promote polymerization, where the yields of poly(MMA) were lower than $6 \%$ within $6 \mathrm{~h}$ (see Table III). GMA can be used as a epoxide and as a monomer. Using GMA as catalytic amount $(0.01 \mathrm{ml})$, the polymerization of MMA afforded poly(MMA) in $42 \%$ yield in diglyme within $6 \mathrm{~h}$. As shown in Figure 1, the yield and molecular weight of the corresponding polymer increased with increasing GMA in the polymerization of MMA. A copolymer containing GMA unit, which was confirmed by ${ }^{1} \mathrm{H}$ NMR, was yielded due to increasing GMA. In this experiment, the concentrations of total monomers increased due to the addition of GMA. Therefore, we also understood that the polymerization takes place in the presence of catalytic amount and relatively large amount of epoxide (GMA).

Reaction temperature also influenced the polymerization of MMA. As shown in Figure 2, the yield of the poly(MMA) increased with arising temperature (30$60^{\circ} \mathrm{C}$ ) suggesting that the polymerization reaction proceeds at relatively higher temperature, e.g., at $60^{\circ} \mathrm{C}$. Triad tacticities of poly(MMA) show that the polymerization is syndiotactic predominant, where $r r$ triad is over $60 \%$. Although the yield of polymer was poor at $30^{\circ} \mathrm{C}$, syndiotactic content at this temperature increased somewhat $(\mathrm{mm} / \mathrm{mr} / \mathrm{rr}=3.0 / 28.5 / 68.5)$ relative to the case $(\mathrm{mm} / \mathrm{mr} / \mathrm{rr}=3.8 / 34.6 / 61.6)$ at $60^{\circ} \mathrm{C}$.

As mentioned above, higher monomer concentration leads to the higher molecular weight polymer. Remembering of this in mind, we compared bulk and solution polymerizations of several monomers such as $n$-BMA, $t$-BMA, c-HMA, $n$-HMA, and MMA as well as BzMA using $\mathrm{CDP} /$ epoxide as pre-initiator. In the bulk polymerizations, molecular weights for all polymethacrylates were extremely high $\left(M_{n}=380 \times 10^{3}-\right.$ $700 \times 10^{3}$ ) compared with polymers obtained from the solution polymerizations (see Figure 3 and Table IV). However, the yields of these polymers were significantly dependent on alkyl substituents on methacrylate. As shown in Figure 3, the yields of polymethacrylates in bulk conditions increased in the following order of alkyl groups: $\mathrm{CH}_{3}<\mathrm{CH}_{2} \mathrm{Ph}, n-\mathrm{C}_{6} \mathrm{H}_{11}<n-\mathrm{C}_{4} \mathrm{H}_{9}, t-\mathrm{C}_{4} \mathrm{H}_{9}$,

Table I. Effects of the concentration of MMA on polymerization ${ }^{\mathrm{a}}$

\begin{tabular}{|c|c|c|c|c|c|c|c|c|}
\hline \multirow{2}{*}{ Entry } & Diglyme & [MMA] & {$[\mathrm{MMA}] /[\mathrm{CDP}]$} & $\mathrm{CHO}$ & Time & Yield & \multirow{2}{*}{$\bar{M}_{n} \times 10^{-3}$} & \multirow{2}{*}{$\bar{M}_{w} / \bar{M}_{n}$} \\
\hline & $\mathrm{ml}$ & $\mathrm{moll}^{-1}$ & molar ratio & $\mathrm{ml}$ & $\mathrm{h}$ & $\%$ & & \\
\hline 1 & 0.5 & 2.6 & $40 / 1$ & 0.5 & 6 & 23 & 101.4 & 2.65 \\
\hline 2 & 0.5 & 5.7 & $160 / 1$ & 0.5 & 6 & 8.9 & 253.7 & 2.86 \\
\hline 3 & 0.5 & 5.7 & $160 / 1$ & 0.5 & 24 & 18 & 504.3 & 2.83 \\
\hline 4 & 2 & 3.6 & $160 / 1$ & 0.5 & 6 & 11 & 232.6 & 2.10 \\
\hline 5 & 10 & 1.2 & $160 / 1$ & 0.5 & 6 & 39 & 26.48 & 1.83 \\
\hline
\end{tabular}

${ }^{a}$ Temperature was set at $60^{\circ} \mathrm{C} ; \mathrm{CHO}=$ cyclohexene oxide. 
Table II. Effects of solvents on polymerization ${ }^{\mathrm{a}}$

\begin{tabular}{clcc}
\hline Entry & Solvent & Monomer & Yield $/ \%$ \\
\hline 1 & DMF & MMA & 80 \\
2 & Diglyme & MMA & 53 \\
3 & Chlorobenzene & MMA & 2.4 \\
4 & Toluene & MMA & Trace \\
5 & CCl & MMA & 0 \\
6 & Xylene & MMA & 0 \\
7 & Acetonitrile & MMA & 0 \\
8 & Acetone & MMA & 0 \\
9 & DMSO $_{10}$ & MMA & 0 \\
10 & DMF $^{\text {b }}$ & EA & 91 \\
11 & DMF $^{\text {b }}$ & $n$-BA & 86 \\
& DMSO $^{\text {b }}$ & $n$-BA & 0 \\
\hline
\end{tabular}

${ }^{\mathrm{a}}$ Monomer, $14.5 \mathrm{mmol}$; CDP, $0.09 \mathrm{mmol}$; solvent, $10.5 \mathrm{ml}$; GMA, $0.01 \mathrm{ml}, 60^{\circ} \mathrm{C}, 24 \mathrm{~h}$. ${ }^{\mathrm{b}} 5.0 \mathrm{ml}$ solvent were used.

Table III. Effects of epoxides on polymerization of $\mathrm{MMA}^{\mathrm{a}}$

\begin{tabular}{clc}
\hline Entry & Epoxides & Yield/\% \\
\hline 1 & GMA & 56.6 \\
2 & CHO & 38.5 \\
3 & Styrene oxide & 49 \\
4 & Chlomethyloxirane & 6.2 \\
5 & Glycidyl phenyl ether & 5.9 \\
6 & 1-Methoxy-2-methylpropylene oxide & 4.8 \\
7 & Propylene oxide & 6.5 \\
\hline
\end{tabular}

${ }^{\mathrm{a}} \mathrm{MMA}, 14.5 \mathrm{mmol}$; CDP, $0.09 \mathrm{mmol}$; diglyme, $10 \mathrm{ml}$; epoxides, $0.5 \mathrm{ml}, 60^{\circ} \mathrm{C}, 6 \mathrm{~h}$.

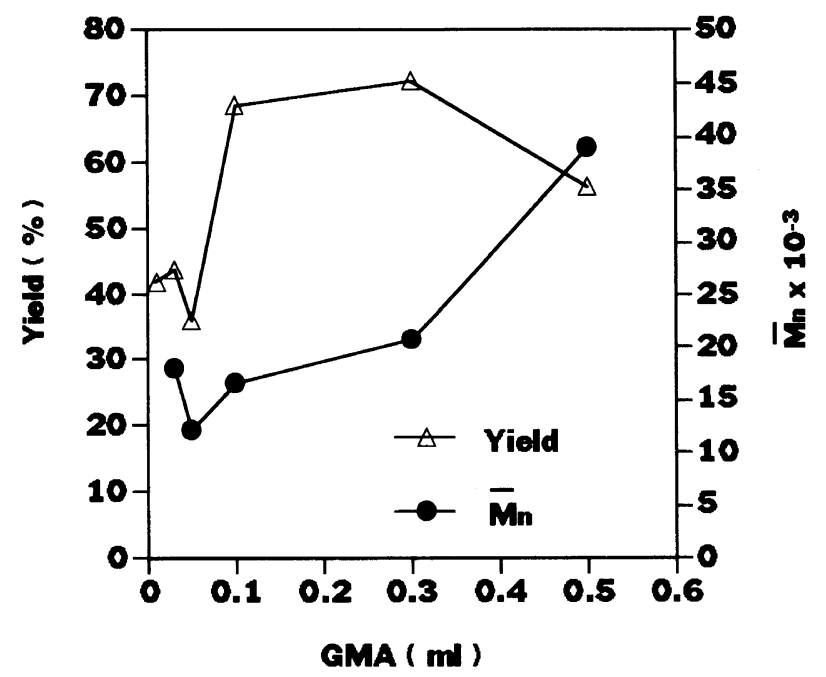

Figure 1. Influence of GMA content on the polymerization of MMA. Conditions: $\mathrm{MMA}=14.5 \mathrm{mmol} ; \mathrm{CDP}=0.09 \mathrm{mmol}$; diglyme $+\mathrm{GMA}=$ $10.5 \mathrm{ml}, 60^{\circ} \mathrm{C}, 6 \mathrm{~h}$.

c- $\mathrm{C}_{6} \mathrm{H}_{11}$. High yields and high molecular weights poly(n-BMA), poly( $t$-BMA), and poly(c-HMA) were easily obtained in the presence of $\mathrm{CDP} /$ epoxide pre-initiator in the bulk conditions. As a control experiment, $n$-BMA was stirred without $\mathrm{CDP} /$ epoxide in bulk at $60^{\circ} \mathrm{C}$ for $24 \mathrm{~h}$, but no polymer was produced.

As can be seen in Figure 3, MMA gave low yield of poly(MMA) but $n$-BMA and $t$-BMA gave high yields of the corresponding polymers in the bulk polymerization conditions. However, copolymerization reactions containing either MMA and $n$-BMA or MMA and $t$-BMA in bulk afforded very poor yields of the corresponding

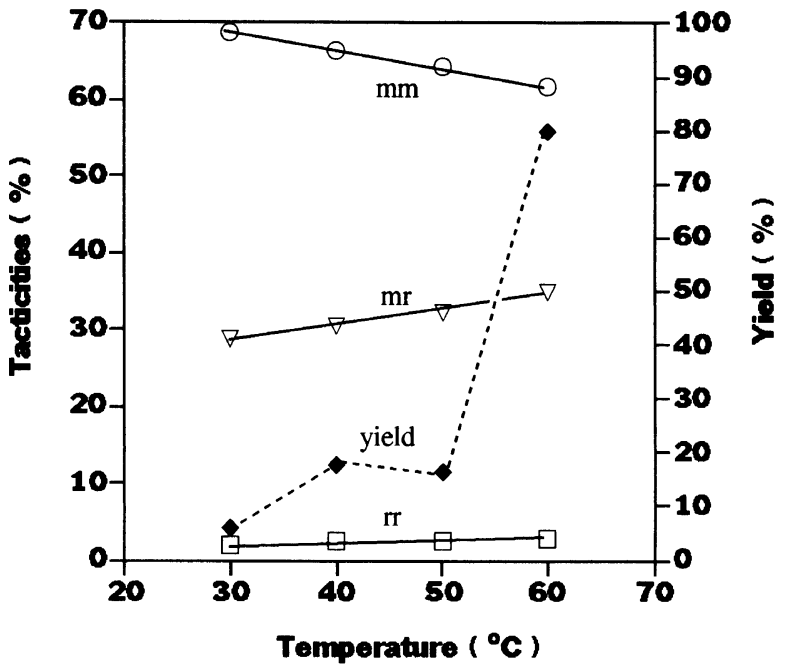

Figure 2. Effects of temperature on polymerization of MMA Conditions: $\mathrm{MMA}=14.5 \mathrm{mmol} ; \mathrm{CDP}=0.09 \mathrm{mmol} ; \mathrm{DMF}=10.5 \mathrm{ml}$; $\mathrm{GMA}=0.01 \mathrm{ml}, 24 \mathrm{~h}$.

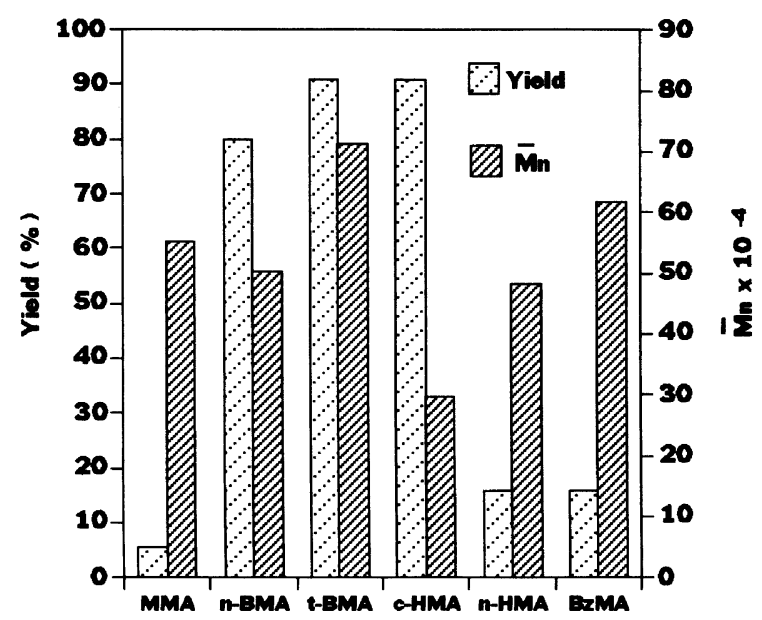

Figure 3. Relations of yield and molecular weight of polymers to monomers. Conditions: monomer $=14.5 \mathrm{mmol}$; $C D P=0.09 \mathrm{mmol}$; epoxide; GMA or $\mathrm{CHO}, 60^{\circ} \mathrm{C}$ for $24 \mathrm{~h}$ in bulk (see Table IV).

copolymers (see entries 19 and 20 in Table IV). The compositions of these two copolymers were determined to be 55/45 ( $n$-BMA/MMA) and 57/43 ( $t$-BMA/MMA), respectively.

MMA and BzMA were polymerized in higher yields in solution polymerization than in bulk condition, but the molecular weights decreased drastically with wide polydispersity in solution polymerization (see entries 15-18 in Table IV). On the other hand, the solution polymerizations of $n$-BMA and $t$-BMA in diglyme resulted in lower molecular weights of $\operatorname{poly}(n$-BMA) and poly(t-BMA), although their yields were not low (see entries 6 and 9 in Table IV). When $n$-BMA was polymerized in non-polar solvent such toluene, poor yield ( $c a .3 \%)$ of poly ( $n$-BMA) was obtained (entry 7 in Table IV). Evidently, the influence of solvent is also significant for the polymerization of $n$-BMA.

In the bulk condition, c-HMA was polymerized with high yield $(91 \%)$ but the molecular weight distribution of this polymer was very wide. In contrast, $n$-HMA resulted in low yield of poly(n-HMA). Furthermore, the polymerization of $n$-BMA and MMA with higher 
Table IV. Polymerization of methacrylates in bulk and solution conditions ${ }^{\mathrm{a}}$

\begin{tabular}{|c|c|c|c|c|c|c|c|}
\hline \multirow{2}{*}{ Entry } & \multirow{2}{*}{ Monomer } & \multirow{2}{*}{ Solvent } & \multicolumn{2}{|l|}{$[\mathrm{M}] /[\mathrm{CDP}]$} & Yield & \multirow{2}{*}{$\bar{M}_{n} \times 10^{-3}$} & \multirow{2}{*}{$\bar{M}_{w} / \bar{M}_{n}$} \\
\hline & & & molar ratio & & $\%$ & & \\
\hline 1 & $n$-BMA & No & $160 / 1$ & GMA & 80 & 502.9 & 1.58 \\
\hline 2 & & No & $320 / 1$ & GMA & 89 & 569.1 & 1.44 \\
\hline 3 & & DMF & $160 / 1$ & $\mathrm{CHO}$ & 21 & 495.2 & 1.49 \\
\hline 4 & & DMF & $320 / 1$ & $\mathrm{CHO}$ & 22 & 589.8 & 1.44 \\
\hline 5 & & DMF & $160 / 1$ & GMA & 21 & 412.2 & 1.42 \\
\hline 6 & & Diglyme & $160 / 1$ & $\mathrm{CHO}$ & 75 & 50.23 & 2.06 \\
\hline 7 & & Toluene & $160 / 1$ & $\mathrm{CHO}$ & 2.2 & - & - \\
\hline 8 & $t$-BMA & No & $160 / 1$ & $\mathrm{CHO}$ & 91 & 711.4 & 1.27 \\
\hline 9 & & Diglyme & $160 / 1$ & $\mathrm{CHO}$ & 45 & 116.2 & 1.43 \\
\hline 10 & c-HMA & No & $160 / 1$ & GMA & 91 & 297.9 & 2.79 \\
\hline 11 & $n$-HMA & No & $160 / 1$ & GMA & 17 & 483.5 & 1.77 \\
\hline 12 & BzMA & No & $240 / 1$ & GMA & 16 & 618.6 & 1.32 \\
\hline 13 & & No & $320 / 1$ & GMA & 14 & 551.7 & 1.47 \\
\hline 14 & & DMF & $160 / 1$ & GMA & 79 & 63.05 & 1.63 \\
\hline 15 & MMA & No & $160 / 1$ & GMA & 5.7 & 387.9 & 1.60 \\
\hline 16 & & No & $320 / 1$ & GMA & 6.8 & 561.7 & 1.27 \\
\hline 17 & & DMF & $160 / 1$ & GMA & 80 & 22.3 & 2.20 \\
\hline 18 & & Diglyme & $160 / 1$ & GMA & 53 & 16.6 & 1.70 \\
\hline 19 & $n$-BMA/MMA & No & $160 / 160 / 1$ & GMA & $3.5^{\mathrm{b}}$ & - & - \\
\hline 20 & $t$-BMA/MMA & No & $160 / 160 / 1$ & $\mathrm{CHO}$ & $7.4^{\mathrm{b}}$ & - & - \\
\hline
\end{tabular}

${ }^{\text {a }} \mathrm{CDP}, 0.09 \mathrm{mmol}$; solvent, $10 \mathrm{ml}$ for $\mathrm{MMA}$ and BzMA, $5 \mathrm{ml}$ for $n$-BMA; epoxide, $0.01 \mathrm{ml}$ for $\mathrm{GMA}, 0.1 \mathrm{ml}$ for $\mathrm{CHO}$; temp $60^{\circ}$; time $24 \mathrm{~h}$ under argone. ${ }^{\mathrm{b}}$ Composition of copolymer: 55/45 (n-BMA/MMA) and 57/43 ( $t$-BMA/MMA).

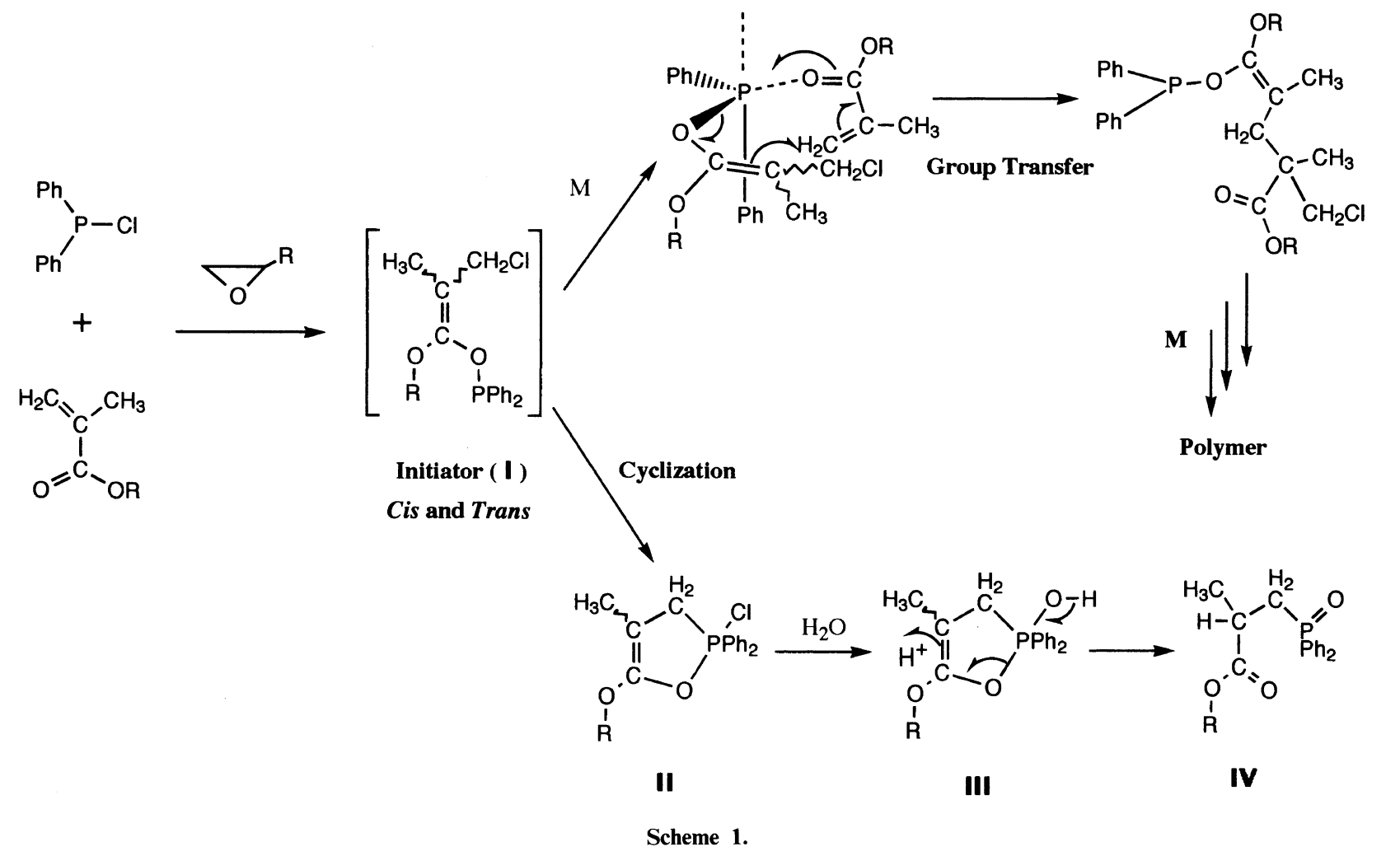

molar ratio of monomer to CDP led to relatively high molecular weight polymer. On comparing the relation between molecular weight and polydispersity, interestingly, we found for all of monomers that the polydispersities of the polymethacrylates became more narrow when molecular weights highly increased. So, it could be concluded that the polymerization of methacrylates with $\mathrm{CDP} /$ epoxide pre-initiator system is especially usable for the synthesis of high molecular weight polymethacrylates.

\section{DISCUSSION}

The above results must be interpreted from the polymerization mechanism. As proposed earlier by us (see Scheme 1), ${ }^{5}$ a mixture containing CDP, epoxide and monomer, firstly, gives an initiator of phosphino enolate (I) which has cis and trans isomers relative to the position between chloromethyl and phosphoxyl groups. In this reaction, epoxide may play a catalytic role. It can be expected that the reaction rate and ratio of isomers for 
the formation of I control the polymerization. From the fact that the isolation of the phosphine oxide $(\mathbf{I V})^{5}$ from the mixture for CDP/monomer, it could be concluded that the cis-I results in IV via intramolecular cyclization route. Thus, prevention of the cyclization of $\mathbf{I}$ is related to enhance a polymerization efficiency. In the bulk polymerization, increment of yield for such monomer having bulky alkyl groups would be due to steric hindrance to the cyclization of cis-I and/or preferential formation of trans-I. The formed trans-I and/or cis-I act as initiators to polymerize methacrylates.

The production of high molecular weight polymethacrylates may be concerned with group transfer polymerization (GTP) mechanism, where the phosphino enolate site on polymeric chain polymerizes monomers one after another without self-quenching like a train transfer reaction or site decomposition. The fact that the higher molecular weight polymers have the more narrow polydispersity would be explained as follows. The propagating chains formed early increase to the extremely extended high polymeric state until the sites of high polymer have no reactivity to the monomer. On the other hand, the relatively small propagating chains induced later react the monomers to reach the high polymeric state. Consequently, the polydispersity narrows in the high polymeric state. The molecular weight of poly $(t-$ BMA) is over seven million with 1.27 polydispersity (see entry 8 in Table IV) after reacted for $24 \mathrm{~h}$. However, the molecular weight of poly( $t$-BMA) obtained within $7 \mathrm{~h}$ under the same conditions was found to be $451.2 \times 10^{3}$ with 1.51 polydispersity. This means that the polydispersity becomes narrow with increment of molecular weight over five million within a long reaction time. From this relation, we consider that the phosphino enolate I forms in the relatively initial state of the polymerization and the propagation reaction follows quickly as I appears. On the other hand, from the effect of monomer concentration on both polymer yield and polymer molecular weight (Table I), we believe that the rate of the propagation reaction is proportional to monomer concentration and is higher than the rate of the formation of I. For this reason, the highly propagated polymer was observed in a relatively shorter reaction time, and the preparation of the high molecular weight of polymethacrylates with high yields took relatively longer reaction time. Poly( $n$-BMA) having ten million (!) magnitude molecular weight with very narrow polydispersity $\left(M_{n}=1.024 \times 10^{6}, M_{w} / M_{n}=1.17\right)$ was prepared by the bulk method in this study. Considering these results, we assume that the propagating site has living character due to no self-quenching although there is no the typical living polymerization process such as rapid disappearance of initiators.

In spite of the low yield for the bulk polymerization of MMA, the molecular weight of poly(MMA) increased to five million with narrow polydispersity. The reason of the low yield for bulk polymerization of MMA would be due to the small substituent of methyl on methacrylate for which either the probability of the formation of cis-I or cyclization of the cis-I become great to reduce initiator efficiency. In the case of using CDP/epoxide as preinitiator, the monomers such as $n$-BMA and $t$-BMA having large alkyl substituents were polymerized nearly quantitatively in bulk conditions. When these monomers were mixed with MMA in the same bulk conditions, however, very poor yields of copolymers were afforded (see entries 19 and 20 in Table IV). This supports that CDP reacts more quickly with MMA than $n$-BMA and $t$-BMA so that the polymerization efficiency decreases drastically due to cyclization of I formed from MMA and CDP. On the other hand, these copolymers compositions by molar ratio were nearly half to half, indicating that the reactivity of the site of propagating chain to monomers MMA and $n$-BMA is nearly identical. So, it can be concluded that the rate of propagation reaction is not dependent on alkyl structures.

Whether polymerization proceeds may be determined by the formation of I. Thus, it is considered that the dependency on temperature, solvents and the epoxides is mainly concerned with the reaction of the formation of the enolate $\mathbf{I}$. The yield of polymer was very poor at lower temperature but high at higher temperature indicating that the formation of enolate $\mathbf{I}$ is temperature dependency (Figure 2). On the other hand, triad tacticity was found to be syndiotactic predominant, and this tendency increased somewhat with reducing temperature. Although higher temperature is an important condition for producing high yield of polymers, the reaction time also influences on polymer molecular weight and polydispersity. In bulk polymerization, elongation of reaction time led to increase molecular weight of the polymer and narrow the polydispersity. Consequently, higher temperature and longer reaction time are required conditions for obtaining controlled polymers in CDP/epoxide system. Although the reason of solvent effect is not clear at present, it is evident that DMF is a suitable solvent in solution polymerization of (meth)acrylates. The CDP/epoxide pre-initiator is also usable in the polymerization of acrylates.

\section{CONCLUSIONS}

The monomers of methacrylates are easily polymerized in the CDP/epoxide pre-initiator system. In these polymerizations, the high molecular weights of polymethacrylates near or over five million can be obtained. This polymerization system has a living character (no self-quenching) although polydispersity cannot be controlled precisely. Considering the simplicity and convenience of the polymerization method, it should be applicable for the preparation of the high molecular weight polymethacrylates. At present, we are developing phosphino enolates initiators to explore a controlled novel polymerization system.

\section{REFERENCES AND NOTES}

1. D. Y. Sogah, W. R. Hertler, O. W. Webster, and G. M. Cohen, Macromolecules, 20, 1473 (1987).

2. H. Yasuda, H. Yamamoto, K. Yokoda, S. Miyake, and A. Nakamura, J. Am. Chem. Soc., 114, 4908 (1992).

3. M. Kuroki, T. Watanabe, T. Aida, and S. Inoue, J. Am. Chem. Soc., 113, 5903 (1991).

4. T. Kitayama, E. Masuda, M. Yamaguchi, T. Nishimura, and K. Hatada, Polym. J., 24, 817 (1992).

5. R. H. Jin and T. Nishikubo, Polymer, 35, 2907 (1994).

6. T. Saegusa, Y. Kimura, N. Ishikawa, and S. Kobayashi, Macro- 
molecules, 9, 724 (1976)

7. S. Kobayashi, S. Lundmark, J. Kadokawa, and A. Albertsson, Macromolecules, 24, 2129 (1991).

8. S. Kobayashi, J. Kadokawa, H. Uyama, and S. Shoda, Polym. J., 23, 817 (1991).

9. F. A. Bovey, "High Resolution NMR of Macromolecules," Academic Press, New York and London, 1976, Chapter X.

10. The structure of $1: 1$ adduct from CDP and epoxide is not quite clear. At least, two isomers $\mathrm{ClCH}_{2} \mathrm{CH}(\mathrm{R})-\mathrm{OPPh}_{2}$ and $\mathrm{CH}_{2}-\mathrm{CH}(\mathrm{R})-\mathrm{O}-\mathrm{P}^{+} \mathrm{Ph}_{2} \mathrm{Cl}^{-}$were speculated. The betaine analo- gous was not isolated because of unstableness in air atmosphere.

11. The structure of $1: 1$ adduct from CDP and vinyl methyl ketone was speculated to be a cyclic enolate, e.g., oxaphospholene $\mathrm{CH}_{2}-\mathrm{CH}=\mathrm{C}\left(\mathrm{CH}_{3}\right)-\mathrm{O}-\mathrm{P}^{+} \mathrm{Ph}_{2} \mathrm{Cl}^{-}$(see ref 12). It cannot be isolated. But, we found that the adduct is turned quickly and quantitatively to a phosphine oxide $\mathrm{Ph}_{2} \mathrm{P}(\mathrm{O}) \mathrm{CH}_{2} \mathrm{CH}_{2} \mathrm{C}(\mathrm{O}) \mathrm{CH}_{3}$ (confirmed by ${ }^{1} \mathrm{H}$ NMR and MS) in air atmosphere.

12. M. Mikolajczyk and A. Zatorski, J. Org. Chem., 56, 1217 (1991). 